\title{
Transcatheter Closure of Patent Ductus Arteriosus
} With Severe Pulmonary Artery Hypertension Through Single Venous Approach: a Propensity Score Match Analysis From Long-term Outcomes

\section{Danqing Hu}

Fujian Medical University

\section{En Chen}

Fujian Medical University Union Hospital

\section{Wei Cai}

Fujian Medical University Union Hospital

Lianglong Chen ( $\square$ lianglongchenfj@126.com )

Fujian Medical University Union Hospital https://orcid.org/0000-0002-9281-6466

\section{Research article}

Keywords: patent ductus arteriosus, pulmonary artery hypertension, transcatheter closure, propensity score matching

Posted Date: May 18th, 2021

DOI: https://doi.org/10.21203/rs.3.rs-524973/v1

License: (c) (1) This work is licensed under a Creative Commons Attribution 4.0 International License. Read Full License 


\section{Abstract}

Background: Up to now, transcatheter closure of patent ductus arteriosus(PDA) without severe pulmonary artery hypertension (PAH) has been well established and accepted in clinical practice. However, in PDA patients with severe $\mathrm{PAH}$, transcatheter closure still remains a challenge, regardless of the tremendous advancement in devices and techniques.

Methods: We reviewed the records of PDA patients who underwent transcatheter device closure though single femoral venous approach between January 2005 and December 2018. Severe PAH was defined as systolic pulmonary artery pressure (SPAP) measured by a catheter $>70 \mathrm{mmHg}$, excluding irreversible severe PAH assessed by trial occlusion. Propensity score matching was used to assemble a cohort of patients with similar baseline characteristics.

Results: A total of 29 patients were in severe PAH (SPAH) group after excluding 2 for failing to the trial occlusion, and 468 in non-severe PAH(non-SPAH) group. After matching, 25 patients were in SPAH group, and 39 in non-SPAH group, without any significant difference in baseline characteristics. The median follow-up periods were 57 and 44 months for groups, respectively. No significant difference was noted in the rates of cumulative adverse events in the 2 groups $(p=0.208)$. No poor effects in left ventricular function and residual shunt evaluated by transthoracic echocardiography(TTE) in SPAH group were detected in the short- and long-term follow-up.

Conclusion: In the patients with PDA associated with reversible severe PAH, compared to non-severe PAH, transcatheter closure through single venous approach has comparable adverse events rates and successful closure rate, with equivalent results of TTE examination at the short- and long-term follow-up.

\section{Introduction}

As pioneered transcatheter closure by Portsmann et al. in $1967^{1}$, the majority of patent ductus arteriosus(PDA) were preferable to accept transcatheter closure for the beneficial outcomes and less invasion ${ }^{2,3}$. The procedure of transcatheter closure usually requires femoral artery puncture, and angiography for duct anatomic and shunting evaluation ${ }^{4}$. However, in several patients without accessible femoral artery, Baykan A et al. had to develop the femoral venous access, with the guidance of transthoracic echocardiography (TTE) and aortogram in the return phase, instead aortic access through femoral artery ${ }^{5}$. Subsequently, accumulating experiences have confirmed that transcatheter closure of PDA though single femoral venous approach is technically feasible, with further simplifying the procedure, and fewer invasions and potential complications ${ }^{6,7}$.

Up to now, transcatheter PDA closure without severe pulmonary artery hypertension (PAH) has been well established and accepted in clinical practice. However, in PDA patients with severe PAH, transcatheter closure still remains a challenge, regardless of the tremendous advancement in devices and techniques. Actually, the reversibility of severe PAH effects the management and prognosis. In the short- and 
intermediate- term of follow-up, several studies had observed partial or complete resolution of PAH after transcatheter closure of PDA patients with severe $\mathrm{PAH}^{8-10}$. However, the results of long-term follow-up are unclear. Furthermore previous studies were single cohort of PDA patients with severe-PAH, without the comparison of clinical outcomes between the severe PAH and non-severe PAH in PDA patients treated by transcatheter closure. Thus, we performed a propensity score matched(PSM) analysis to evaluate the safety and efficacy of transcatheter closure of PDA patients with severe PAH though single femoral venous approach from the long-term outcomes.

\section{Materials And Methods}

\section{Patient population}

We reviewed the records of PDA patients who had underwent transcatheter device closure though single femoral venous approach at Fujian Medical University Union Hospital, between January 2005 and December 2018. These patients all had clinical and echocardiographic findings of PDA, and severe PAH was defined as systolic pulmonary artery pressure (SPAP) measured by a catheter $>70 \mathrm{mmHg}$. The PDA patients with irreversible severe $\mathrm{PAH}$, assessed by trial occlusion, were excluded ${ }^{10}$. Meanwhile the exclusion criteria also contained the unavailable follow-up information(less than one year). The study was approved by the ethics committee of Fujian Medical University Union Hospital and the informed consent was also waived for the retrospective nature(2020KY021).

Patients' records routinely contain demographics, clinical manifestations, patient weight, TTE results, PDA size at its narrowest point, PAH before and immediately after occluder release, device type and device size. The anatomy of PDA was classified following Krichenko's classification by TTE ${ }^{11}$.

Additionally, TTE was performed to evaluate the degree of the residual shunt (RS) after the device release. A left-to-right shunt without a jet was defined as trivial RS, with a jet diameter $<2 \mathrm{~mm}$ as a small shunt and with a jet $>2 \mathrm{~mm}$ in diameter as large shunt ${ }^{3}$. Device protrusion was described as any visible protruding of the device into the descending aorta or pulmonary artery, based on TTE.

\section{Interventional procedure}

Infants and young children received general anesthesia before operation (usually under 10 years older), and local anesthesia for adults and well-coordinated children. Meanwhile, the procedures were carried out by experienced operators. The TTE was performed to define the size and anatomy of PDA before procedure in cardiac catheterization laboratory. $6 \mathrm{~F}$ radial sheath was placed in the femoral vein before administration of heparin $(100 \mathrm{U} / \mathrm{kg}) .6 \mathrm{~F}$ multipurpose catheter (Cordis) was implanted through the inferior caval vein, right atrium, right ventricle and main pulmonary artery (IVC-RA-RV-PA) to accomplish the complete hemodynamic evaluation. Subsequently, the MP catheter was advanced through the PDA into the descending aorta. With the replacement of guidewire $(260 \mathrm{~cm} \times 0.035 \mathrm{inch})$, the MP catheter was exchanged for deliver sheaths. The occluder device in suitable size was selected according to the weight 
of patients and anatomy of PDA measured by TTE. The occluder device was deployed following the standard protocol but without descending aortography.

For PDA patient with severe PAH, trial occlusion was performed for 30 minutes to record the change of hemodynamic and clinical data after the complete hemodynamic evaluation and anatomy of PDA measured by TTE. The occluder would be released if a fall in pulmonary artery pressure or no elevation, no decrease in aortic pressure, and no worsening of signs and symptoms ${ }^{10}$. Otherwise, the occluding device would be retracted into the delivery sheath for the irreversible PAH. A repeat TTE was executed 10 min after device release to assess the degree of the RS, and to make sure the device in appropriate position without protrusion or iatrogenic aorta coarctation.

\section{Statistical analyses}

Continuous variables are expressed as mean \pm standard deviation(SD). Chi-square test for categorical variables and t-test for continuous variables were involved in the statistical analyses. P-value $<0.05$ was considered statistical significance. For the pretty intergroup differences between severe PAH(SPAH) and non-severe PAH(non-SPAH) groups in baseline characteristics (Table 1), propensity similar score matching was performed to identify a cohort patient with comparable baseline characteristics. 1:2 matching protocol without replacement (greedy-matching algorithm) with a caliper width equal to 0.2 of the SD of the logit of the propensity score was used to match. In order to evaluate the discrepancy before and after matching, standardized differences were estimated for all the baseline covariates and $<10 \%$ for the given covariate indicate a relatively small imbalance. All datum was analyzed by the SPSS 24.0 software package (SPSS Inc., Chicago, IL, USA). 
Table 1

Baseline characteristics between SPAH and non-SPAH groups before and after propensity score matching

Characteristics

Male/female, $n$

Age (years),

median(interquartile range)

\begin{tabular}{|c|c|c|c|c|c|c|}
\hline Male/female, $n$ & $8 / 21$ & $119 / 349$ & 0.796 & $7 / 18$ & $6 / 33$ & 0.221 \\
\hline \multirow{2}{*}{$\begin{array}{l}\text { Age (years), } \\
\text { median(interquartile range) }\end{array}$} & 30.00 & 6.50 & \multirow[t]{2}{*}{0.004} & 31.00 & 29.00 & \multirow[t]{2}{*}{0.794} \\
\hline & $\begin{array}{l}(14.50- \\
37.50)\end{array}$ & $\begin{array}{l}(2.93- \\
31.75)\end{array}$ & & $\begin{array}{l}(14.50- \\
38.00)\end{array}$ & $\begin{array}{l}(10.00- \\
44.00)\end{array}$ & \\
\hline Height $(\mathrm{cm})$, mean \pm SD & $\begin{array}{l}145.67 \pm \\
29.57\end{array}$ & $\begin{array}{l}122.78 \pm \\
36.16\end{array}$ & $<0.001$ & $\begin{array}{l}145.00 \\
\pm 30.26\end{array}$ & $\begin{array}{l}146.03 \pm \\
27.06\end{array}$ & 0.888 \\
\hline $\begin{array}{l}\text { Body weight }(\mathrm{kg}) \text {, mean } \pm \\
\text { SD }\end{array}$ & $\begin{array}{l}39.98 \pm \\
16.71\end{array}$ & $\begin{array}{l}32.36 \pm \\
21.32\end{array}$ & 0.025 & $\begin{array}{l}39.58 \pm \\
16.67\end{array}$ & $\begin{array}{l}41.60 \pm \\
16.70\end{array}$ & 0.637 \\
\hline $\mathrm{SBP}(\mathrm{mmHg})$, mean $\pm \mathrm{SD}$ & $\begin{array}{l}112.00 \pm \\
18.29\end{array}$ & $\begin{array}{l}106.81 \pm \\
17.32\end{array}$ & 0.119 & $\begin{array}{l}113.08 \\
\pm 18.76\end{array}$ & $\begin{array}{l}117.69 \pm \\
16.83\end{array}$ & 0.311 \\
\hline $\mathrm{DBP}(\mathrm{mmHg})$, mean $\pm \mathrm{SD}$ & $\begin{array}{l}64.45 \pm \\
10.12\end{array}$ & $\begin{array}{l}64.39 \pm \\
10.27\end{array}$ & 0.974 & $\begin{array}{l}63.08 \pm \\
9.16\end{array}$ & $\begin{array}{l}65.00 \pm \\
10.21\end{array}$ & 0.448 \\
\hline $\operatorname{LVED}(\mathrm{mm})$, mean $\pm \mathrm{SD}$ & $\begin{array}{l}55.62 \pm \\
14.70\end{array}$ & $\begin{array}{l}45.65 \pm \\
12.01\end{array}$ & $<0.001$ & $\begin{array}{l}56.85 \pm \\
15.00\end{array}$ & $\begin{array}{l}57.13 \pm \\
13.95\end{array}$ & 0.934 \\
\hline $\operatorname{LVES}(\mathrm{mm})$, mean $\pm \mathrm{SD}$ & $\begin{array}{l}36.15 \pm \\
12.57\end{array}$ & $28.74 \pm 8.76$ & $<0.001$ & $\begin{array}{l}36.96 \pm \\
13.09\end{array}$ & $\begin{array}{l}37.87 \pm \\
10.81\end{array}$ & 0.761 \\
\hline $\mathrm{LA}(\mathrm{mm})$, mean $\pm \mathrm{SD}$ & $\begin{array}{l}35.21 \pm \\
11.82\end{array}$ & $27.92 \pm 9.44$ & $<0.001$ & $\begin{array}{l}36.09 \pm \\
12.40\end{array}$ & $\begin{array}{l}36.10 \pm \\
10.63\end{array}$ & 0.999 \\
\hline $\mathrm{AO}(\mathrm{mm})$, mean $\pm \mathrm{SD}$ & $\begin{array}{l}28.29 \pm \\
6.29\end{array}$ & $\begin{array}{l}21.71 \pm \\
12.50\end{array}$ & 0.005 & $\begin{array}{l}27.81 \pm \\
6.08\end{array}$ & $\begin{array}{l}26.77 \pm \\
7.29\end{array}$ & 0.557 \\
\hline $\mathrm{PDA} / \mathrm{AO}$, mean $\pm \mathrm{SD}$ & $\begin{array}{l}0.32 \pm \\
0.13\end{array}$ & $0.22 \pm 0.10$ & $<0.001$ & $\begin{array}{l}0.30 \pm \\
0.11\end{array}$ & $\begin{array}{l}0.30 \pm \\
0.15\end{array}$ & 0.996 \\
\hline $\operatorname{LVEF}(\%)$, mean \pm SD & $\begin{array}{l}62.54 \pm \\
11.42\end{array}$ & $67.18 \pm 7.22$ & 0.039 & $\begin{array}{l}62.72 \pm \\
11.58\end{array}$ & $\begin{array}{l}61.46 \pm \\
11.18\end{array}$ & 0.665 \\
\hline $\mathrm{PDA}(\mathrm{mm})$, mean $\pm \mathrm{SD}$ & $\begin{array}{l}9.01 \pm \\
4.03\end{array}$ & $4.43 \pm 1.97$ & $<0.001$ & $\begin{array}{l}8.00 \pm \\
3.03\end{array}$ & $\begin{array}{l}7.52 \pm \\
3.25\end{array}$ & 0.552 \\
\hline $\mathrm{PDA}(\mathrm{mm})$, range & $3.4-20.0$ & $1.0-14.7$ & & $\begin{array}{l}3.4- \\
14.7\end{array}$ & $3.0-14.7$ & \\
\hline
\end{tabular}

Before matching

SPAH non-SPAH

$(n=29)$

$(n=468)$

$119 / 349$

6.50

0.796

0.004

P-
value

$8 / 21$

30.00

$(14.50-$

$145.67 \pm$

$39.98 \pm$

$112.00 \pm \quad 106.81 \pm$

$64.39 \pm$

$45.65 \pm$

After matching

SPAH
$(n=25)$

$7 / 18$

$6 / 33$

0.221

$(n=39)$

non-

P-

value

(n)

29.00

0.794

31.00

$(14.50$

38.00)

(10.00)

.

SBP: systolic blood pressure,DBP: diastolic blood pressure, LVED: left ventricular end diastolic , LVES: left ventricular end systolic, LA: left atrium, AO: Aorta, LVEF: left ventricular eject fraction 


\begin{tabular}{|c|c|c|c|c|c|c|}
\hline \multirow[t]{2}{*}{ Characteristics } & \multicolumn{3}{|c|}{ Before matching } & \multicolumn{3}{|c|}{ After matching } \\
\hline & $\begin{array}{l}\text { SPAH } \\
(n=29)\end{array}$ & $\begin{array}{l}\text { non-SPAH } \\
(n=468)\end{array}$ & $\begin{array}{l}\mathrm{P} \text { - } \\
\text { value }\end{array}$ & $\begin{array}{l}\text { SPAH } \\
(n=25)\end{array}$ & $\begin{array}{l}\text { non- } \\
\text { SPAH } \\
(n=39)\end{array}$ & $\begin{array}{l}\mathrm{P} \text { - } \\
\text { value }\end{array}$ \\
\hline $\begin{array}{l}\text { PDA anatomy } \\
\text { (tuber/funnel/window, n) }\end{array}$ & 19/9/1 & $309 / 144 / 15$ & 0.997 & $17 / 8 / 0$ & $23 / 10 / 6$ & 0.119 \\
\hline $\begin{array}{l}\text { Shunt } \\
\text { (Left to right/bi-directional, } \\
\text { n) }\end{array}$ & $23 / 6$ & $466 / 2$ & $<0.001$ & $21 / 4$ & $38 / 1$ & 0.051 \\
\hline \multicolumn{7}{|c|}{ SBP: systolic blood pressure,DBP: diastolic blood pressure, LVED: left ventricular end diastolic , } \\
\hline LVES: left ventricular end sy & c, LA: I & um, AO: Aorta & EF & & fros & \\
\hline
\end{tabular}

\section{Results}

\section{Baseline characteristics}

A total of 499 patients' records from 2005 to 2018 with available datum had been reviewed. Of these, 468 were in non-severe PAH(non-SPAH) group with 154 normal pulmonary artery pressure(PAP), 314 mildmoderate PAP, and 29 were in severe PAH(SPAH) group after excluding 2 for failing to the trial occlusion. All the included PDA patients had successfully underwent transcatheter closure with Cardi-O-Fix (Starway Medical Technology, China) and Taraxaci (Shandong Visee Medical Devices Co., China).

Prior to the PSM, there were significant discrepancies between the two groups in age, height, body weight, left ventricular end diastolic (LVED), left ventricular end systolic(LVES), left atrium(LA), Aorta(AO), left ventricular eject fraction(LVEF), and PDA size (Supplementary Table 1) at its narrowest point(Table 1). Hence, we used sex, blood pressure and these above as covariates to match SPAH( severe PAH) and nonSPAH groups. After matching, 25 patients were in SPAH group, and 39 patients in non-SPAH group, without any significant difference in baseline characteristics.

Interestingly, the anatomies of PDA between the two groups were comparative no matter before or after matching, including the types of tuber, funnel and window, Meanwhile, the bi-directional shunt in SPAH group was not more prominent before and after matching.

\section{Procedural characteristics}

SPAP and mean pulmonary artery pressure (MPAP) measured by a catheter before and after occlusion in SPAH group were more higher than non-SPAH group, which was also not balanced by PSM(Table 2). For local anesthesia and occluder size, the SPAH group were more dominant before matching, but after matching, they were equivalent(Table 2). 
Table 2

Procedure characteristics between SPAH and non-SPAH groups before and after propensity score matching

\begin{tabular}{|c|c|c|c|c|c|c|c|}
\hline \multicolumn{2}{|l|}{ Characteristics } & \multicolumn{3}{|c|}{ Before matching } & \multicolumn{3}{|c|}{ After matching } \\
\hline & & $\begin{array}{l}\text { SPAH } \\
(n=29)\end{array}$ & $\begin{array}{l}\text { non- } \\
\text { SPAH } \\
(n=468)\end{array}$ & $\begin{array}{l}\mathrm{P} \text { - } \\
\text { value }\end{array}$ & $\begin{array}{l}\text { SPAH } \\
(n=25)\end{array}$ & $\begin{array}{l}\text { non- } \\
\text { SPAH } \\
(n=39)\end{array}$ & $\begin{array}{l}\mathrm{P}- \\
\text { value }\end{array}$ \\
\hline \multicolumn{2}{|c|}{$\begin{array}{l}\text { General anesthesia/ } \\
\text { local anesthesia }\end{array}$} & $5 / 29$ & $247 / 468$ & $<0.001$ & $4 / 21$ & $10 / 29$ & 0.363 \\
\hline \multirow[t]{2}{*}{ PAPS $(m m H g)$} & $\begin{array}{l}\text { before } \\
\text { operation }\end{array}$ & $\begin{array}{l}99.21 \pm \\
24.85\end{array}$ & $\begin{array}{l}30.34 \pm \\
11.47\end{array}$ & $<0.001$ & $\begin{array}{l}101.95 \pm \\
26.18\end{array}$ & $\begin{array}{l}34.64 \pm \\
14.2\end{array}$ & $<0.001$ \\
\hline & $\begin{array}{l}\text { post } \\
\text { operation }\end{array}$ & $\begin{array}{l}56.95 \pm \\
19.82\end{array}$ & $\begin{array}{l}24.16 \pm \\
5.80\end{array}$ & $<0.001$ & $\begin{array}{l}64.28 \pm \\
15.74\end{array}$ & $\begin{array}{l}20.30 \pm \\
11.01\end{array}$ & $<0.001$ \\
\hline \multirow[t]{2}{*}{ PAPM(mmHg) } & $\begin{array}{l}\text { before } \\
\text { operation }\end{array}$ & $\begin{array}{l}63.73 \pm \\
14.86\end{array}$ & $\begin{array}{l}18.52 \pm \\
8.53\end{array}$ & $<0.001$ & $\begin{array}{l}64.28 \pm \\
15.74\end{array}$ & $\begin{array}{l}20.30 \pm \\
11.01\end{array}$ & $<0.001$ \\
\hline & $\begin{array}{l}\text { post } \\
\text { operation }\end{array}$ & $\begin{array}{l}35.68 \pm \\
12.64\end{array}$ & $\begin{array}{l}15.06 \pm \\
4.11\end{array}$ & $<0.001$ & $\begin{array}{l}36.00 \pm \\
13.65\end{array}$ & $\begin{array}{l}14.23 \pm \\
6.14\end{array}$ & $<0.001$ \\
\hline \multicolumn{2}{|c|}{$\operatorname{LVED}(\mathrm{mm})$, mean $\pm \mathrm{SD}$} & $\begin{array}{l}52.54 \pm \\
13.09\end{array}$ & $\begin{array}{l}41.82 \pm \\
10.57\end{array}$ & $<0.001$ & $\begin{array}{l}53.47 \pm \\
13.73\end{array}$ & $\begin{array}{l}52.25 \pm \\
13.54\end{array}$ & 0.753 \\
\hline \multicolumn{2}{|c|}{$\operatorname{LVES}(\mathrm{mm})$, mean $\pm \mathrm{SD}$} & $\begin{array}{l}36.96 \pm \\
11.85\end{array}$ & $\begin{array}{l}27.74 \pm \\
8.51\end{array}$ & $<0.001$ & $\begin{array}{l}37.77 \pm \\
12.56\end{array}$ & $\begin{array}{l}37.85 \pm \\
12.62\end{array}$ & 0.982 \\
\hline \multicolumn{2}{|c|}{$\mathrm{LA}(\mathrm{mm})$, mean $\pm \mathrm{SD}$} & $\begin{array}{l}32.20 \pm \\
9.14\end{array}$ & $\begin{array}{l}25.26 \pm \\
8.31\end{array}$ & $<0.001$ & $\begin{array}{l}32.67 \pm \\
10.04\end{array}$ & $\begin{array}{l}31.75 \pm \\
9.70\end{array}$ & 0.744 \\
\hline \multicolumn{2}{|c|}{$\mathrm{AO}(\mathrm{mm})$, mean $\pm \mathrm{SD}$} & $\begin{array}{l}28.03 \pm \\
7.21\end{array}$ & $\begin{array}{l}21.17 \pm \\
6.89\end{array}$ & $<0.001$ & $\begin{array}{l}27.45 \pm \\
7.30\end{array}$ & $\begin{array}{l}27.23 \pm \\
6.44\end{array}$ & 0.907 \\
\hline \multicolumn{2}{|c|}{$\operatorname{LVEF}(\%)$, mean \pm SD } & $\begin{array}{l}57.79 \pm \\
10.36\end{array}$ & $\begin{array}{l}63.03 \pm \\
9.01\end{array}$ & 0.01 & $\begin{array}{l}57.55 \pm \\
10.84\end{array}$ & $\begin{array}{l}54.34 \pm \\
11.49\end{array}$ & 0.340 \\
\hline \multicolumn{2}{|c|}{ Residual shunt, $\mathrm{n}$} & 4 & 28 & 0.016 & 4 & 3 & 0.444 \\
\hline \multicolumn{2}{|l|}{ trivial shunt } & 0 & 10 & & 0 & 1 & \\
\hline \multicolumn{2}{|c|}{ small shunt (<2mm) } & 2 & 14 & & 2 & 1 & \\
\hline \multicolumn{2}{|c|}{ large shunt ( $\geq 2 \mathrm{~mm}$ ) } & 2 & 4 & & 2 & 1 & \\
\hline \multicolumn{8}{|c|}{$\begin{array}{l}\text { PASP: pulmonary arterial systolic pressure, PAPM: mean pulmonary artery pressure, LVED : left } \\
\text { ventricular end diastolic, }\end{array}$} \\
\hline
\end{tabular}


The median follow-up periods were 57 and 44 months for groups, respectively. There was no difference in follow-up time between the SPAH and non-SPAH groups. During this follow-up period, no any device migration, embolization, arrhythmia, hemolysis, vascular access and massive blood loss had occurred. No adverse clinical events between the two groups was detected in the short-term follow-up(less than one month), but with one death respectively between the two groups in the long-term follow-up(more than one year). No significant difference was observed in adverse clinical events between the two groups after matching $(p=0.208)$.

For echocardiograph examination, before matching, SPAH group was more worse than non-SPAH group no matter short- or long-term follow-up. However after matching, no significant difference between the two groups was observed, such as LVED, LVES, LA, AO and LVEF in the short- and long-term follow-up.

Before matching, RS was detected in 4 patients in the SPAH group in the short-term follow-up ( 2 small and 2 large), but at the last follow-up, only 1 trivial RS in the SPAH group. Meanwhile in the non-SPAH group, RS was present in 28 patients in the short-term follow-up (10 trivial, 14 small and 4 large), whereas at the last follow-up, only 2 small RS in the non-SPAH group. After matching, no discrepancy in the rate of RS was detected(Table 3). 
Table 3

Follow-up characteristics between SPAH and non-SPAH groups before and after propensity score matching

\begin{tabular}{|c|c|c|c|c|c|c|}
\hline \multirow[t]{2}{*}{ Characteristics } & \multicolumn{3}{|c|}{ Before matching } & \multicolumn{3}{|c|}{ After matching } \\
\hline & $\begin{array}{l}\text { SPAH } \\
(n=29)\end{array}$ & $\begin{array}{l}\text { non-SPAH } \\
(n=468)\end{array}$ & $\begin{array}{l}\mathrm{P} \text { - } \\
\text { value }\end{array}$ & $\begin{array}{l}\text { SPAH } \\
(n=25)\end{array}$ & $\begin{array}{l}\text { non-SPAH } \\
(n=39)\end{array}$ & $\begin{array}{l}\mathrm{P}- \\
\text { value }\end{array}$ \\
\hline $\begin{array}{l}\text { Clinical follow-up time, } \\
\text { median (interquartile } \\
\text { range) }\end{array}$ & $\begin{array}{l}1700(662- \\
3028)\end{array}$ & $\begin{array}{l}\text { 1318(836- } \\
1941)\end{array}$ & 0.108 & $\begin{array}{l}\text { 1062(615- } \\
2707)\end{array}$ & $\begin{array}{l}1536(901- \\
1941)\end{array}$ & 0.568 \\
\hline Death, $n$ & 1 & 1 & 0.008 & 1 & 0 & 0.208 \\
\hline $\begin{array}{l}\text { Echo follow-up time, } \\
\text { median(interquartile } \\
\text { range) }\end{array}$ & $\begin{array}{l}1678(784- \\
2533)\end{array}$ & $\begin{array}{l}924(498- \\
1566)\end{array}$ & 0.003 & $\begin{array}{l}1380(526- \\
2108)\end{array}$ & $\begin{array}{l}896(410- \\
1550)\end{array}$ & 0.133 \\
\hline $\operatorname{LVED}(\mathrm{mm})$, mean $\pm \mathrm{SD}$ & $\begin{array}{l}48.34 \pm \\
10.93\end{array}$ & $\begin{array}{l}40.31 \pm \\
9.12\end{array}$ & $<0.001$ & $\begin{array}{l}48.43 \pm \\
11.32\end{array}$ & $\begin{array}{l}46.10 \pm \\
10.42\end{array}$ & 0.44 \\
\hline $\operatorname{LVES}(\mathrm{mm})$, mean $\pm \mathrm{SD}$ & $\begin{array}{l}32.84 \pm \\
10.39\end{array}$ & $\begin{array}{l}25.60 \pm \\
6.99\end{array}$ & 0.001 & $\begin{array}{l}33.06 \pm \\
11.06\end{array}$ & $\begin{array}{l}30.86 \pm \\
9.64\end{array}$ & 0.419 \\
\hline $\mathrm{LA}(\mathrm{mm})$, mean $\pm \mathrm{SD}$ & $\begin{array}{l}32.05 \pm \\
10.82\end{array}$ & $\begin{array}{l}26.14 \pm \\
9.75\end{array}$ & 0.002 & $\begin{array}{l}32.25 \pm \\
11.69\end{array}$ & $\begin{array}{l}29.79 \pm \\
8.37\end{array}$ & 0.341 \\
\hline $\mathrm{AO}(\mathrm{mm})$, mean $\pm \mathrm{SD}$ & $\begin{array}{l}27.75 \pm \\
6.13\end{array}$ & $\begin{array}{l}21.74 \pm \\
6.64\end{array}$ & $<0.001$ & $\begin{array}{l}27.10 \pm \\
5.84\end{array}$ & $\begin{array}{l}26.90 \pm \\
6.46\end{array}$ & 0.898 \\
\hline $\operatorname{LVEF}(\%)$, mean \pm SD & $\begin{array}{l}60.40 \pm \\
12.21\end{array}$ & $\begin{array}{l}66.62 \pm \\
7.80\end{array}$ & 0.016 & $\begin{array}{l}60.05 \pm \\
13.24\end{array}$ & $\begin{array}{l}62.70 \pm \\
10.59\end{array}$ & 0.41 \\
\hline Residual shunt & 1 & 2 & $<0.001$ & 1 & 1 & 0.747 \\
\hline trivial shunt & 1 & 0 & & 1 & 1 & \\
\hline small shunt(<2mm) & 0 & 2 & & 0 & 0 & \\
\hline large shunt ( $\geq 2 \mathrm{~mm}$ ) & 0 & 0 & & 0 & 0 & \\
\hline
\end{tabular}

\section{Discussion}

The propensity score matched analysis was performed to assess the safety and efficacy of transcatheter closure of PDA with severe PAH though single femoral venous approach, and we found that device occlusion in patients with severe PAH (excluding the irreversible severe PAH by temporary occlusion in cath lab) only by femoral venous routine demonstrated comparable adverse events rates and successful 
closure rate, with equivalent results of TTE examination at the short- and long-term follow-up to patients with non-severe PAH.

Indeed, previous studies had preliminary investigated the efficacy of transcatheter closure of PDA with severe PAH, and indicated that this approach would be safe, feasible and effective. As Yan et al. reported, a total of 20 PDA adult patients with severe PAH had successfully accomplished device occlusions, with the dimensions of left atrium, left ventricle and pulmonary artery increased considerably in 3-6 months of follow-up ${ }^{10}$. And Zhang et al. had investigated the immediate and two-year follow up effects of transcatheter closure of 29 adult patients with severe PAH, without any death or major complication 9 . During the follow-up period, Zhang et al. also observed left ventricular function usually recovered within a few months. Controversially, ISHWARAPPA et al had found that device embolization in aorta was higher in PDA patients with severe PAH from 6-9 months, which had included adults and infants.

In fact, these previous studies were small size in included patients with limited time of follow-up, and only observed single cohort of PDA patients with severe-PAH from clinical outcomes and TTE results. Moreover, the definition of severe PAH based upon the mean PAP $>45 \mathrm{mmHg}$ or $>50 \mathrm{mmHg}$. For the inherit detects of previous reports, we included PDA patients with severe PAH upon SPAP $>70 \mathrm{mmHg}$ by a catheter, whose mean PAH was $63.73 \pm 14.86 \mathrm{mmHg}$ (Table 2). And the time of follow-up in our study including the clinical adverse events and TTE results were pretty longer than previous studies(Table 3 ). In order to determine the safety and efficacy of PDA patients with severe PAH by transcatheter closure, we compared these severe $\mathrm{PAH}$ patients to non-severe PAH patients, which had widely accepted transcatheter closure as the first-choice treatment of PDA.

What's more, in our present study, we only included PDA patients though single femoral venous approach. As Liu et al. had revealed that replacing the arterial approach by single venous access had the potential to eliminate almost complications associated with arterial puncture, such as occlusion, embolism, dissection, bleeding and pseudoaneurysm formation ${ }^{4}$. If transcatheter closure was an inevitable compromise to PDA, the only we could choose was the comparable effects but with fewer invasions and potential complication, even providing more possibility to helpless patients, just like severe PAH. Hence, we conducted this study to seek the foundation of evidence-based medicine in transcatheter closure of PDA patients with severe PAH only by single venous approach. According to clinical experience, skillful echocardiographer was able to evaluate the blood flow in the aorta and pulmonary arteries and describe the anatomy of PDA. Distinguished with the study by Liu et al. ${ }^{4}$, the single venous approach was completely in the guidance of TTE, without aortogram in the return phase in our study. And we had confirmed the feasible and safety of this method in PDA patients regardless of the level of PAP. Besides the reduction of contrast medium during procedure, an additional positive effect of venous approach was to decrease the time of fluoroscopy and hospital stay ${ }^{4,12}$.

As we all known, the PDA patients with severe PAH were pretty different to non-severe PAH in the baseline characteristics. Severe PAH patients were generally associated with poorer left ventricular function and large PDA, which was also consistent with our study(Table 1). However, after matching with these 
relevant characteristics between SPAH and non-SPAH groups, they were equivalent. Actually, the severe $\mathrm{PAH}$ in our study was selected, only including reversible severe PAH. And the preoperative evaluation criteria of severe PAH reversibility was temporary occlusion by occluder in our medical center, which had been considered more accurate than calculation of pulmonary vascular resistance ${ }^{10,13}$.

In this study, we described the first comparison between severe PAH and non-severe PAH in PDA patients treated by transcatheter closure. Surprisingly, the effects of transcatheter closure by single venous approach were comparable between the two groups not matter before or after matching, especially with equivalent cardiac function and incidence of death. This would remind us to perform transcatheter assessment firstly, and temporary occlusion if necessary for all PDA patients, which could avoid the reversible severe $\mathrm{PAH}$ absent.

Our study had several limitation. First, this research was retrospective, not randomized. Although we had performed PSM to balance the baseline characteristics, the inherent limitation of a retrospective study was still unavoidable. Second, the sample size of severe PAH was limited, and the PAH was not assessed again by catheterization during the period of follow-up. Therefore, further researches with large sample size, prospection and randomization are necessary.

\section{Conclusion}

In the patients with PDA associated with reversible severe PAH, compared to non-severe PAH, transcatheter closure through single venous approach has comparable adverse events rates and successful closure rate, with equivalent results of TTE examination at the short- and long-term follow-up. Transcatheter closure of PDA with severe PAH through single venous approach would be reasonable, and trial occlusion would be necessary to avoid the reversible severe PAH absent. Further research with large sample size is requirement.

\section{Abbreviations}

PDA: patent ductus arteriosus

TTE: transthoracic echocardiography

PAH: pulmonary artery hypertension

PSM: propensity score matched

SPAP: systolic pulmonary artery pressure

RS: residual shunt

SD: standard deviation 
LVED: left ventricular end diastolic

LVES: left ventricular end systolic

LA: left atrium

AO: Aorta

LVEF: left ventricular eject fraction

\section{Declarations}

\section{Statement on ethics approval and consent}

The study was approved by the ethics committee of Fujian Medical University Union Hospital and the informed consent was also waived for the retrospective nature(2020KY021).

\section{Consent for publication}

Not applicable.

\section{Availability data and materials}

All data generated or analyzed during this study are included in this published article and in the supplementary information files.

\section{Competing interests}

All of the authors declare that there is no conflict of interest in this article.

\section{Funding}

This study was mainly supported by Startup Fund for scientific research, Fujian Medical University(Grant number: 2019QH1054) and partially sponsored by Fujian provincial health technology project(Grant number: 2020QNA035) .

\section{Authors' contributions}

Lianglong Chen designed the study and provided administrative support. Danqing Hu and En Chen participated in the data analysis and drafted the manuscript. Wei Cai participated in data collection. All authors read and approved the final manuscript.

\section{Acknowledgements}

Not applicable. 


\section{References}

1. Porstmann W, Wierny L, Warnke H.Closure of persistent ductus arteriosus without thoracotomy. Ger Med Mon. 1967;12:259-261.

2. Djer MM, Saputro DD, Putra ST, Idris NS. Transcatheter closure of patent ductus arteriosus: 11 years of clinical experience in Cipto Mangunkusumo Hospital, Jakarta, Indonesia. Pediatr Cardiol. 2015;36:1070-1074.

3. Jin M, Liang YM, Wang XF, Guo BJ, Zheng K, Gu Y, Lyu ZY.A Retrospective Study of 1,526 Cases of Transcatheter Occlusion of Patent Ductus Arteriosus. Chin Med J (Engl). 2015;128:2284-2289.

4. Liu J, Gao L, Tan HL, Zheng QH, Liu L, Wang Z.Transcatheter closure through single venous approach for young children with patent ductus arteriosus: A retrospective study of 686 cases. Medicine (Baltimore). 2018;97:e11958.

5. Baykan A, Narin N, Ozyurt A, Argun M, Pamukcu O, Onan SH, Sezer S, Baykan Z, Uzum K.Do we need a femoral artery route for transvenous PDA closure in children with ADO-I? Anatol J Cardiol. 2015;15:242-247.

6. Zahn EM, Nevin P, Simmons C, Garg R.A novel technique for transcatheter patent ductus arteriosus closure in extremely preterm infants using commercially available technology. Catheter Cardiovasc Interv. 2015;85:240-248.

7. Chen W, Yan X, Huang Y, Sun X, Zhong L, Li J, Chen H, Zhong H, Chen L.Transthoracic echocardiography as an alternative major guidance to angiography during transcatheter closure of patent ductus arteriosus: technical feasibility and clinical relevance. Pediatr Cardiol. 2015;36:14-19.

8. Bhalgat PS, Pinto R, Dalvi BV.Transcatheter closure of large patent ductus arteriosus with severe pulmonary arterial hypertension: Short and intermediate term results. Ann Pediatr Cardiol. 2012;5:135-140.

9. Zhang CJ, Huang YG, Huang XS, Huang T, Huang WH, Xia CL, Mo YJ.Transcatheter closure of large patent ductus arteriosus with severe pulmonary arterial hypertension in adults: immediate and twoyear follow-up results. Chin Med J (Engl). 2012;125:3844-3850.

10. Yan C, Zhao S, Jiang S, Xu Z, Huang L, Zheng H, Ling J, Wang C, Wu W, Hu H, Zhang G, Ye Z, Wang H.Transcatheter closure of patent ductus arteriosus with severe pulmonary arterial hypertension in adults. Heart. 2007;93:514-518.

11. Krichenko A, Benson LN, Burrows P, Moes CA, McLaughlin P, Freedom RM.Angiographic classification of the isolated, persistently patent ductus arteriosus and implications for percutaneous catheter occlusion. Am J Cardiol. 1989;63:877-880.

12. Baykan A, Narin N, Özyurt A, Argun M, Pamukçu Ö, Onan SH, Sezer S, Baykan Z, Üzüm K.Do we need a femoral artery route for transvenous PDA closure in children with ADO-I? Anatol J Cardiol. 2015;15:242-247.

13. Thanopoulos BD, Tsaousis GS, Djukic M, Al Hakim F, Eleftherakis NG, Simeunovic SD.Transcatheter closure of high pulmonary artery pressure persistent ductus arteriosus with the Amplatzer muscular 
ventricular septal defect occluder. Heart. 2002;87:260-263.

\section{Supplementary Files}

This is a list of supplementary files associated with this preprint. Click to download.

- supplementarytable1.docx 